\section{T cells get a ride}

Strategies that inhibit the immune checkpoint blockade (ICB) and reactivate $\mathrm{T}$ cells using monoclonal antibodies against programmed cell death protein 1 (PD1) and PD1 ligand 1 (PDL1) have been used to successfully treat immunogenic tumours, and recent efforts have focused on combining this approach with other therapies to maximize its potential. Now, two articles published in Science Translational Medicine - describe the positive effects of combining the inhibition of angiogenesis and ICB. On the one hand, angiogenesis inhibitors can facilitate the access of cytotoxic T cells (CTLs) to tumours, and on the other hand, checkpoint inhibitors counteract the effect of antiangiogenic therapy of promoting immune evasion and $\mathrm{T}$ cell exhaustion.

Dysfunctional tumour blood vessels can limit the extravasation and effectiveness of T cells, so enhancing vascular normalization may improve the efficacy of cancer immunotherapies. To test this hypothesis, De Palma and colleagues evaluated the effects of the bispecific antibody A2V - which targets angiopoietin 2 (ANG2) and vascular endothelial growth factor A (VEGFA) - in six mouse models that included metastatic breast cancer (MMTV-PyMT), RIP1-Tag2 pancreatic neuroendocrine tumour (RT2-PNET), colon cancer and melanoma. Treatment with $\mathrm{A} 2 \mathrm{~V}$ inhibited tumour growth, decreased metastasis formation and improved survival more efficiently than blocking each target individually. A2V promoted vascular normalization, assessed by increased blood vessel coverage by pericytes, and facilitated the extravasation and recruitment of interferon- $\gamma(\mathrm{IFN} \gamma)$-activated CD8 ${ }^{+}$CTLs.
Surprisingly, some of the antitumour effects of A2V were CTLmediated, as they could be neutralized by $\mathrm{T}$ cell depletion. Combined blockade of ANG2 and VEGFA with A2V increased immunogenicity in certain tumours by increasing antigen crosspresentation and the phagocytic activity of tumour-associated antigen-presenting cells. Further experiments showed that CTLs concurrently upregulated the expression of PDL1 in tumour cells, which suggests that A2V could trigger immune activation as well as an immunosuppressive response in the tumour. Inhibition of IFN $\gamma$ or PDL1 improved the antitumour activity of $\mathrm{A} 2 \mathrm{~V}$, especially in the colon cancer model.

Bergers and colleagues also observed an upregulation of PDL1 by IFN $\gamma$-expressing T cells during treatment with the VEGF receptor (VEGFR) inhibitors sorafenib and DC101 in RT2-PNET, MMTV-PyMT and glioblastoma (GBM) mouse models, so they hypothesized that inhibition of PDL1 could prolong the antitumour response during anti-angiogenic therapy. RT2-PNET mice treated with DC101 antibodies showed a decrease in tumour burden; however, these tumours became refractory over time. Combination of anti-VEGFR2 and anti-PDL1 therapies extended the survival of RT2-PNET and MMTV-PyMT mice compared with single-agent treatment, but this combination showed no significant added benefit in the GBM model.

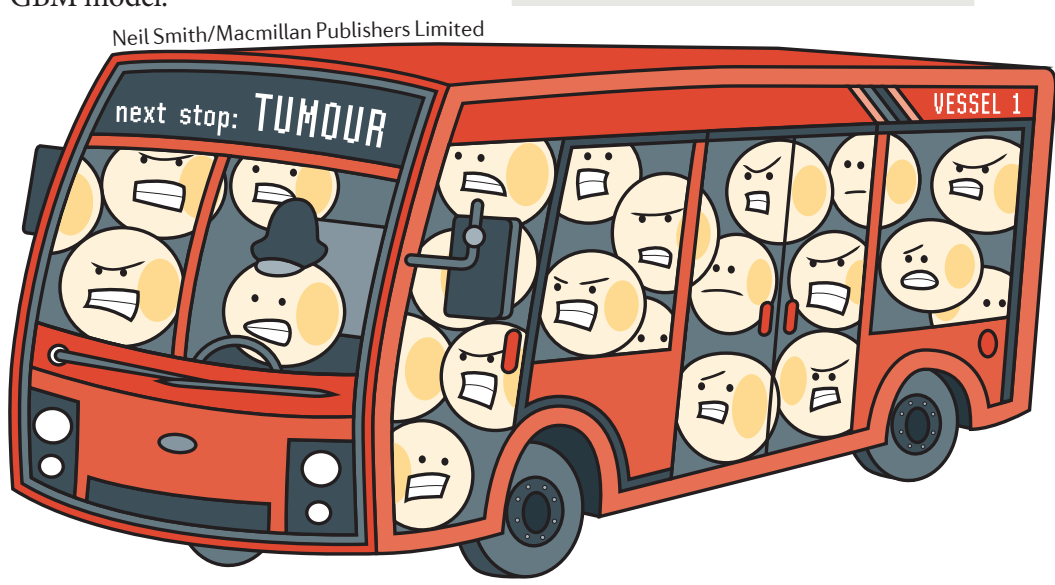

What is the mechanism behind this synergy? Notably, combining anti-VEGFR and anti-PDL1 antibodies induced high endothelial venules (HEVs) in the RT2-PNET and MMTV-PyMT models but not in the GBM model. These HEVs promoted lymphocyte infiltration and activity through activation of lymphotoxin- $\beta$ receptor (LT $\beta R$ ) signalling. Direct activation of LT $\beta R$ with an agonistic antibody - CBE11 - in combination with anti-angiogenic immunotherapy enhanced HEV formation, the immune response, apoptosis and necrosis in RT2-PNET, MMTV-PyMT and GBM models. Together, these findings suggest that the anti-angiogenic immunotherapy failed in the treatment of GBM owing to the lack of HEV formation, which limited T cell infiltration.

These two papers add to the growing research on effective ways of combining immunotherapies with different targeted therapies to improve both efficacy and delivery of these drugs as well as opening new possibilities of exploring vessel normalization in cancer treatment.

M. Teresa Villanueva, Senior Editor Nature Reviews Drug Discovery

This article originally appeared in Nat. Rev. Drug Discov. (doi:10.1038/nrd.2017.103).

ORIGINAL ARTICLES Schmittnaegel, M. et al. Dual angiopoietin-2 and VEGFA inhibition elicits antitumor immunity that is enhanced by PD-1 checkpoint blockade. Sci. Transl Med. 9, eaak9670 (2017) I Allen, E. et al. Combined antiangiogenic and anti-PD-L1 therapy stimulates tumor immunity through HEV formation. Sci. Transl Med. 9, eaak9679 (2017) 